\title{
Les aquarelles de Vincent Fossat : L'art au service de la science ${ }^{1}$
}

\section{The Watercolors of Vincent Fossat: Art in the Service of Science}

\author{
John R. Dolan ${ }^{1}$ \\ ${ }^{1}$ Sorbonne Université CNRS, Laboratoire d'Océanographie de Villefranche-sur-Mer, Station Zoologique, 06230 \\ Villefranche-sur-Mer, France, john.dolan@imev-mer.fr
}

\begin{abstract}
RÉSUMÉ. Vincent Fossat (1822-1891) peintre naturaliste niçois a travaillé avec le biologiste Jean-Baptiste Barla pendant de nombreuses années. V. Fossat a ainsi produit de remarquables aquarelles de champignons, de fleurs et de poissons. Les peintures servaient de documentation aux spécimens étudiés par J-B Barla, enregistrant leurs couleurs et leurs aspects vivants. II a également réalisé des lithographies pour les publications de J-B Barla sur les champignons et les orchidées. Les magnifiques aquarelles détaillées de V. Fossat ont été conservées au Musée d'Histoire Naturelle de Nice et présentées périodiquement dans des expositions locales, mais elles sont peu connues en dehors de Nice. Nous nous efforçons ici de faire connaître Vincent Fossat et ses aquarelles à un large public. Un bref historique de la vie privée et artistique de Vincent Fossat est présenté et des exemples d'aquarelles de Fossat sont donnés.

MOTS-CLÉS. Illustration scientifique, biologie naturaliste au 19e siècle, mycologie, botanique, ichtyologie.
\end{abstract}

Vincent Fossat (1822-1891) a produit des centaines de magnifiques aquarelles sur des sujets d'histoire naturelle - champignons, plantes à fleurs et poissons. Ses peintures détaillées tenaient lieu d'enregistrement des sujets vivants, un peu comme les photographies aujourd'hui. Elles ont été réalisées pour son employeur de longue date, Jean-Baptiste Barla, le célèbre naturaliste de Nice. En 1896, ce dernier a légué ses aquarelles, parmi ses donations substantielles au Muséum d'Histoire Naturelle de Nice, qu'il avait dirigé pendant de nombreuses années. Longtemps oubliées, elles furent « redécouvertes » et portées à la connaissance du public par Eugène Caziot lorsqu'il prit la direction du Muséum d'Histoire Naturelle de Nice en 1912: elles étaient rangées dans des boîtes dans le grenier du musée. Les aquarelles de Fossat, jamais été exposées de son vivant, ont été reconnues comme un bien précieux par le Muséum (par exemple Isnard \& Cordier 1935), et en l'honneur de son travail artistique, une rue de Nice a été baptisée de son nom en 1923 (Anon, 1923). Les aquarelles ont été périodiquement exposées, mais uniquement à Nice et dans les villes environnantes. Un numéro spécial des Annales du Muséum d'Histoire Naturelle de Nice publié en 1996 ( $\left.{ }^{\circ} 11\right)$, consacré à Jean-Baptiste Barla, en contient une douzaine de reproductions. Plusieurs d'entre elles ont été reproduites : dans un livre sur les peintres de la région niçoise (Delfaÿ 1998), un livre sur les premiers naturalistes de Nice (Fredj \& Meinardi 2007) et un ouvrage richement illustré sur les fonds du Muséum d'Histoire Naturelle de Nice (Chamagne Rollier \& Defä̈ 2013). Cependant, ces publications, toutes en français, sont épuisées et les bibliothèques du monde entier n'en contiennent que quelques dizaines.

Il est intéressant de noter que, ces dernières années, certains biologistes ont utilisé les représentations de Fossat pour leur objectif initial, à savoir montrer à quoi ressemblaient les organismes vivants. La figure 1 montre une peinture de Fossat représentant un poisson d'eau profonde qui figure en bonne place sur la couverture d'un livre sur les écosystèmes d'eau profonde de la Méditerranée; plusieurs autres de ses illustrations de poissons d'eau profonde apparaissent dans le texte (WWF/IUCN 2004), et une aquarelle de Fossat représentant un champignon est utilisée

\footnotetext{
${ }^{1}$ Traduction de Marie-Christine Maurel.
} 
comme illustration de couverture pour un article concernant les études de Barla sur les champignons (Cuccuini 2009). Cependant, les aquarelles de Vincent Fossat sont largement inconnues du grand public. Notre objectif est ici de fournir une introduction aux aquarelles de Vincent Fossat. On donnera tout d'abord une brève esquisse de sa vie en se basant largement sur les récits figurant dans Defaÿ (1998) et Chamagne Rollier \& Defaÿ (2013) ainsi que sur les communications personnelles de Jöelle Defaÿ. Une sélection de ses aquarelles suivra pour mettre en valeur les qualités et l'étendue de ses illustrations scientifiques.

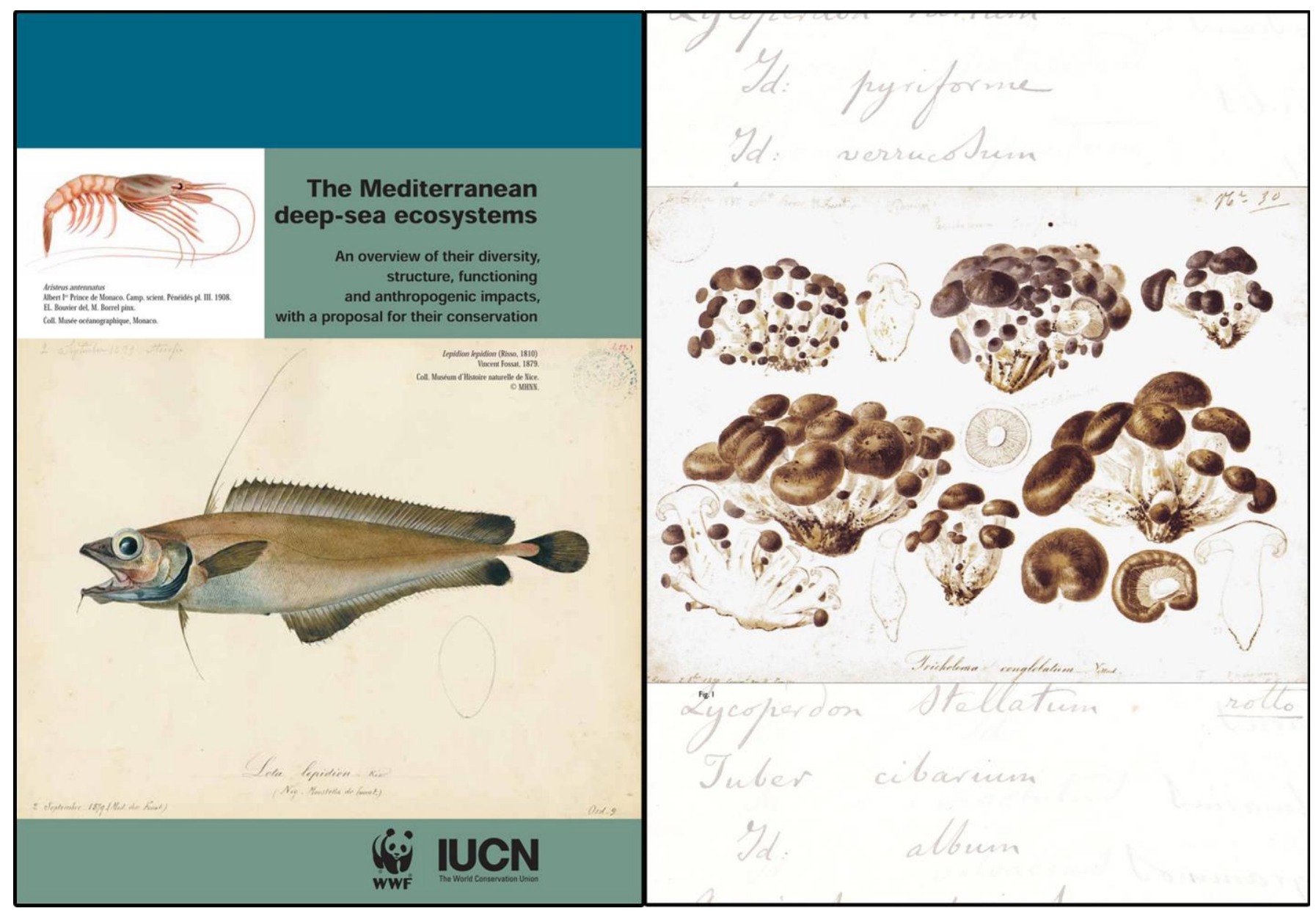

Fig. 1. une peinture de Fossat représentant un poisson d'eau profonde sur la couverture d'un livre sur les écosystèmes d'eau profonde de la Méditerranée , et une aquarelle de Fossat représentant un champignon est utilisée comme illustration de couverture pour un article concernant les études de Barla sur les champignons.

\section{La vie privée et artistique de Vincent Fossat}

On sait très peu de choses sur la vie privée de Fossat. Un court récit, fondé sur les archives municipales (Beltrutti 1996), fournit les seules informations disponibles. Les archives indiquent que Vincent Etienne Fossat (1822-1891) appartenait à une grande famille niçoise. Il est le $7^{\text {ème }}$ de 9 enfants, nés en succession rapide entre 1814 et 1827 d'Antoine Fossat et de Camille Gras. Vincent Fossat est orphelin à l'âge de 20 ans, et est enregistré comme employé de maison en 1842. Il a épousé Marie-Thérèse Bouis (date de mariage inconnue) et a eu deux enfants, une fille Anne, née en 1853, et un fils, Fortuné né en 1854. Il est décédé à l'âge de 69 ans en 1891 laissant derrière lui sa femme, sa fille, son fils et son employeur Jean-Baptiste Barla. Si nous savons peu de choses sur sa vie privée, nous connaissons encore moins avec certitude sa formation d'artiste.

Eugène Caziot, qui a redécouvert les aquarelles de Vincent Fossat, affirme que celui-ci a été formé par Louis Garneray (1783-1857), un peintre du $19^{\text {ème }}$ siècle connu pour ses représentations de scènes marines, notamment de batailles maritimes (Caziot 1918). Malheureusement, Caziot n'a 
fourni aucune source pour son affirmation. S'il existe des preuves documentaires que Garneray a effectivement passé des hivers à Nice à partir de 1841 (Fighiera 1912), les activités de Garneray à Nice n'ont fait l'objet d'aucun compte rendu. L'affirmation séduisante, mais sans fondement, de Caziot selon laquelle Fossat aurait reçu l'enseignement d'un maitre des scènes marines, a été reprise dans des récits ultérieurs de la vie de Fossat (Delfaÿ 1998 ; Fredj \& Gaston 2007 ; Monat 2011 ; Chamagne Rollier \& Defaÿ 2013).

Indépendamment de la manière dont il s'est formé ou du lieu où il l'a fait, la première œuvre d'art datée de Fossat démontre indiscutablement qu'il était, à l'âge de 29 ans, un artiste accompli. Il s'agit d'une lithographie datée de 1850, qui représente une scène de bord de mer à Nice. L'œuvre, "Le Quai du Midi et les Ponchettes » (Fig. 2), est conservée au Musée Masséna de Nice. Le Musée Masséna possède aujourd'hui 32 œuvres de Fossat et il est regrettable qu'aucune ne soit exposée. Il s'agit surtout d'aquarelles mais il y a aussi des dessins et des lithographies. Malheureusement, seules 7 des 32 œuvres sont datées, allant de 1850 à 1880, et peu sont signées. De nombreuses aquarelles montrent cependant le talent évident de Fossat pour l'aquarelle, par exemple la figure 3.

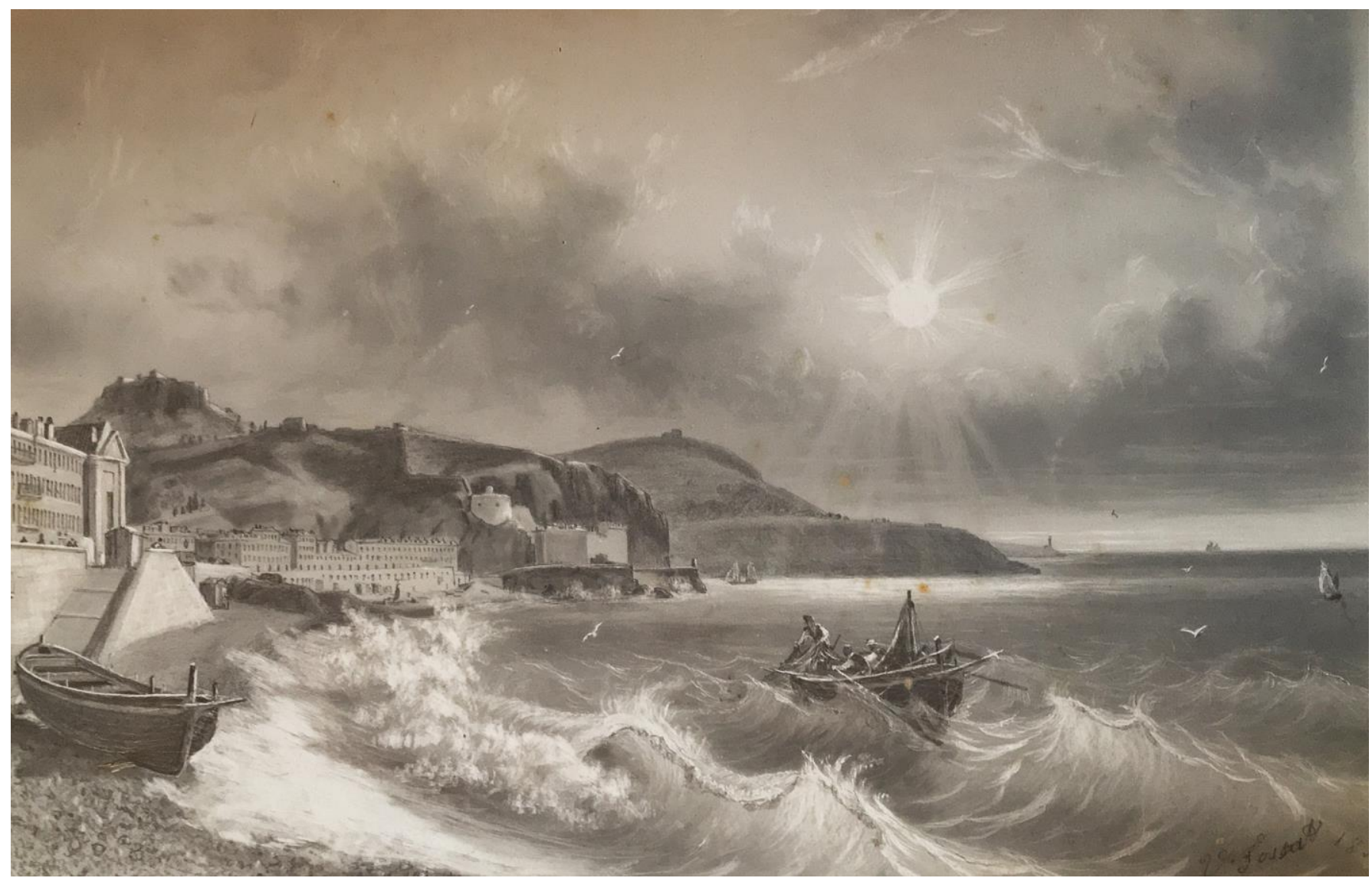

Fig. 2. Le Quai du Midi et les Ponchettes. La taille de l'original : 18,5 x 27,5 cm. Le lithographe est catalogué par le Musée Masséna (Nice) "lithographie rehaussée à la mine de plomb à divers endroits", Ville de Nice, Musée Masséna MAH-9201. 


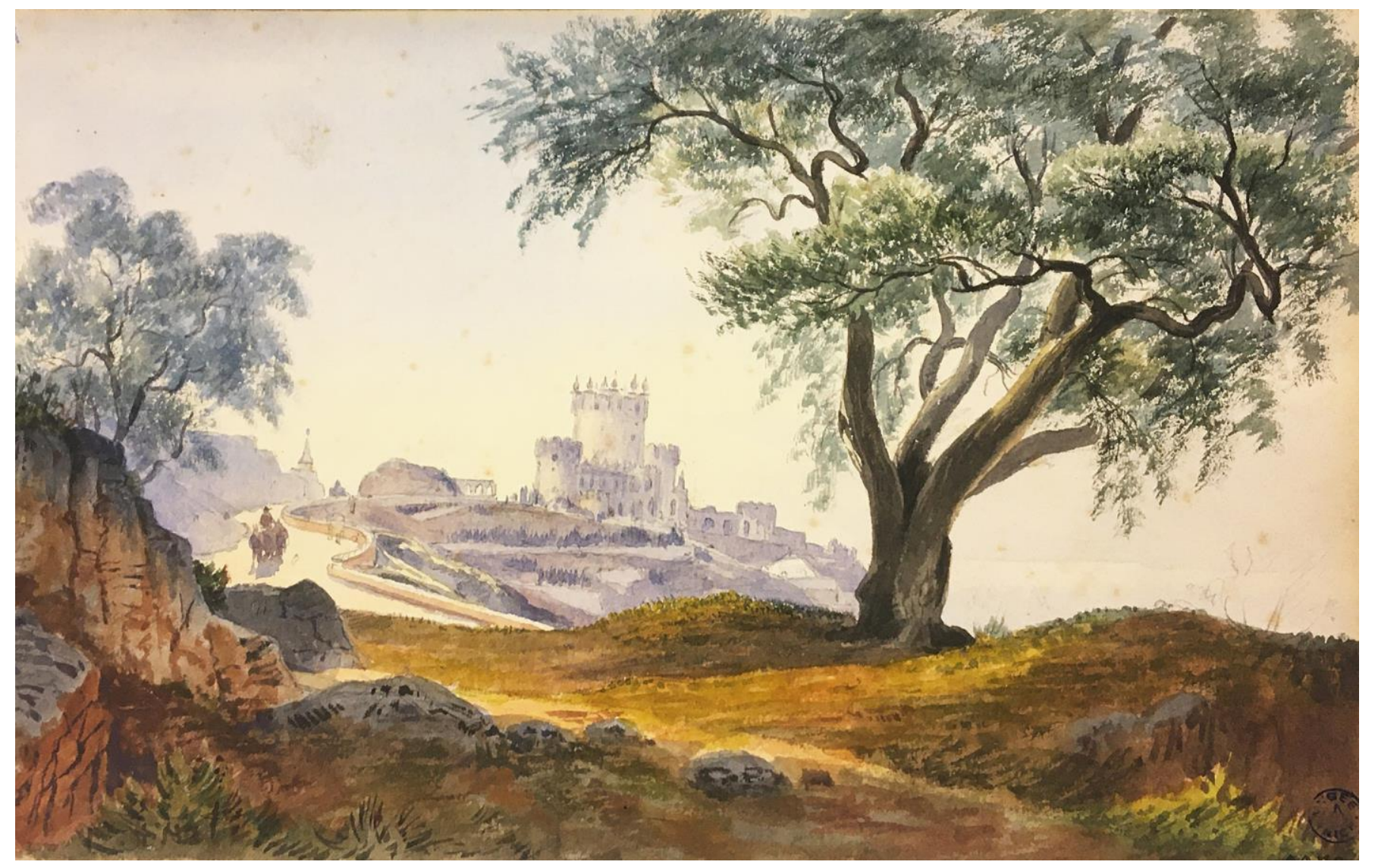

Fig. 3. "Le Château de l'Anglais (1856)" La taille de l'original : 13,3 x 20,7 cm. Ville de Nice, Musée Masséna, MAH-3270.

En 1853, Jean-Baptiste Barla, qui était déjà un naturaliste accompli, engagea Vincent Fossat comme illustrateur scientifique (Trimbach 1996). Selon Trimbach, (dont le récit se fonde sur la correspondance de Barla), le lithographe Xavier Caillol, qui avait réalisé quelques lithographies pour Barla lui-même, a recommandé Fossat à Barla. Vincent Fossat est resté au service de Barla jusqu'à la fin de sa vie, travaillant comme lithographe, illustrateur et peintre de modèles en plâtre de champignons. Au cours des nombreuses années où ils ont travaillé ensemble, les deux hommes ont noué un lien indéfectible. Comme l'a fait remarquer Jöelle Defaÿ (1998), Barla n'aurait pas l'héritage scientifique qu'il possède aujourd'hui sans les contributions artistiques de Fossat, et Fossat n'aurait probablement pas pu vivre en tant qu'artiste sans le soutien de Barla.

Jean-Baptiste Barla est né en 1817 à Nice, quelques années seulement avant Vincent Fossat, et comme ce dernier, dans une famille de plusieurs enfants. Cependant, la famille de Barla était d'un niveau de vie bien différent de celle de Fossat. Le père et le grand-père de Barla étaient des marchands prospères, des «capitaines de commerce », qui achetaient, transportaient et vendaient une variété de marchandises allant de la morue salée et de l'huile d'olive aux graines (Beltrutti 1996). En tant que seul héritier mâle, il était destiné à reprendre l'entreprise familiale et il a reçu une éducation classique dès son plus jeune âge. Il a également bénéficié d'une grande liberté pour s'adonner à l'histoire naturelle, principalement aux champignons. En 1851, à l'âge de 24 ans, il hérite de l'entreprise familiale et de biens totalisant 278000 «livres » (Fredj \& Meinhardi 2007), soit, en monnaie actuelle, plusieurs millions d'euros. Barla est alors en mesure, non seulement de se consacrer à ses activités d'histoire naturelle, mais aussi d'employer d'autres personnes pour l'aider dans ses efforts. C'est ainsi qu'il a pu vouer sa vie et sa fortune à l'histoire naturelle. Barla devient directeur du Muséum d'Histoire Naturelle de Nice en 1862. Il succède à Jean-Baptiste Vernay qui avait fondé le Muséum, le tout premier musée de Nice en 1846 (Thevenon 1990).

Barla engage Vincent Fossat en 1853. Au début, Fossat s'est joint à d'autres pour peindre, en couleurs naturelles, les nouveaux moules en plâtre de champignons que Barla avait mis au point 
pour créer des spécimens permanents, à l'échelle réelle et plus vrais que nature, des champignons qu'il collectait. Les aquarelles de Fossat représentant des spécimens fraîchement récoltés servaient à montrer aux peintres les couleurs du spécimen vivant. Cependant, les talents de lithographe de Fossat ont rapidement été exploités.

La première monographie illustrée de Barla sur les champignons paraît en 1859, «Les Champignons de la Province de Nice et Principalement les Especes Comestibles, Suspectes ou Vénéneuses Dessinés d'Apres Nature et Décrits ». Elle est illustrée de 48 lithographies, dont 45 sont de Fossat. Toutes les monographies ultérieures de Barla seront principalement illustrées par les lithographies de Fossat. Au total, les publications de Barla (Barla 1859, 1868, 1876, 1888, 1889, 1890, 1892) contiennent 172 lithographies, et Fossat en a réalisé 168. Pour 31 de ces lithographies, Fossat est crédité en tant que seul auteur de l'illustration originale et de la lithographie. La figure 4 montre un exemple d'aquarelle de Fossat, la pierre lithographique sur laquelle il a dessiné des éléments de l'aquarelle, et la planche finale, imprimée et colorée à la main. Il est remarquable que tous ces éléments aient été conservés au Muséum d'Histoire Naturelle de Nice.

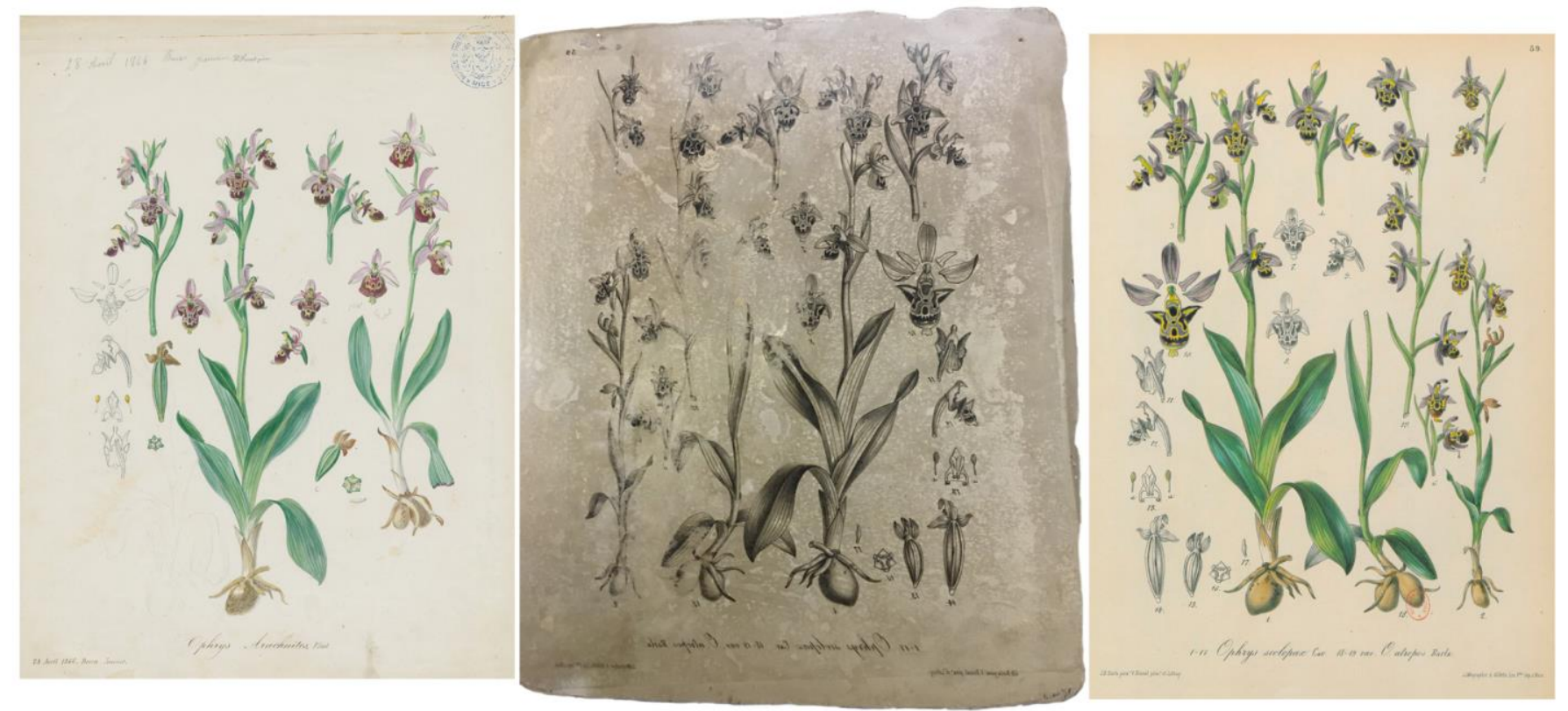

Fig. 4. Un exemple d'aquarelle de Fossat, la pierre lithographique avec des éléments d'aquarelle, et la planche finale, planche 59, dans le livre de Barla (Barla 1868)

Il semble que, pour Barla, les aquarelles de Fossat étaient destinées à enregistrer les aspects vivants des organismes et à être utilisées pour produire des images, comme dans les lithographies, ou des modèles pour guider la peinture des plâtres de champignons. Environ 835 de ses aquarelles sont conservées au Muséum d'Histoire Naturelle de Nice et inventoriées sur le site «Joconde » du Ministère français de la Culture :

https://www.pop.culture.gouv.fr/search/list?base $=\% 5 \mathrm{~B} \% 22 \mathrm{Collections} \% 20 \mathrm{des} \% 20 \mathrm{mus} \% \mathrm{C} 3 \% \mathrm{~A} 9$ es $\% 20 \mathrm{de} \% 20 \mathrm{France} \% 20 \% 28 \mathrm{Joconde} \% 29 \% 22 \% 5 \mathrm{D} \&$ mainSearch $=\% 22$ Vincent $\% 20 \mathrm{Fossat} \% 22$

Si l'on examine la production d'aquarelles de Fossat année par année, on voit que les sujets des aquarelles de Fossat ont varié avec le temps, correspondant probablement aux changements d'intérêts de Barla (Fig. 5). Fossat a peint des champignons tout au long de son séjour chez Barla, mais en nombre relativement restreint. Il a réalisé un total de 133 peintures de champignons sur une période de 35 ans. Les plantes à fleurs ont été son sujet de la fin des années 1860 au début des années 1870, ce qui correspond à peu près à l'époque de la monographie de Barla sur les orchidées (Barla, 1868). Curieusement, la plupart des aquarelles de Fossat représentent des poissons de la mer près de Nice. Elles constituent le plus grand nombre d'œuvres (plus de 300) et ont été produites de la 
fin des années 1870 au début des années 1880, mais n'ont été utilisées dans aucune des publications de Barla, lequel n'a jamais publié sur les poissons. On ne peut que supposer que Barla avait en tête un projet sur les poissons à la fin des années 1870, mais qu'il a ensuite abandonné ce sujet pour revenir à son thème favori, les champignons. Il se peut aussi que Barla ait simplement laissé à Fossat la liberté de choisir ses propres sujets et que Fossat soit revenu à son premier sujet, la mer.

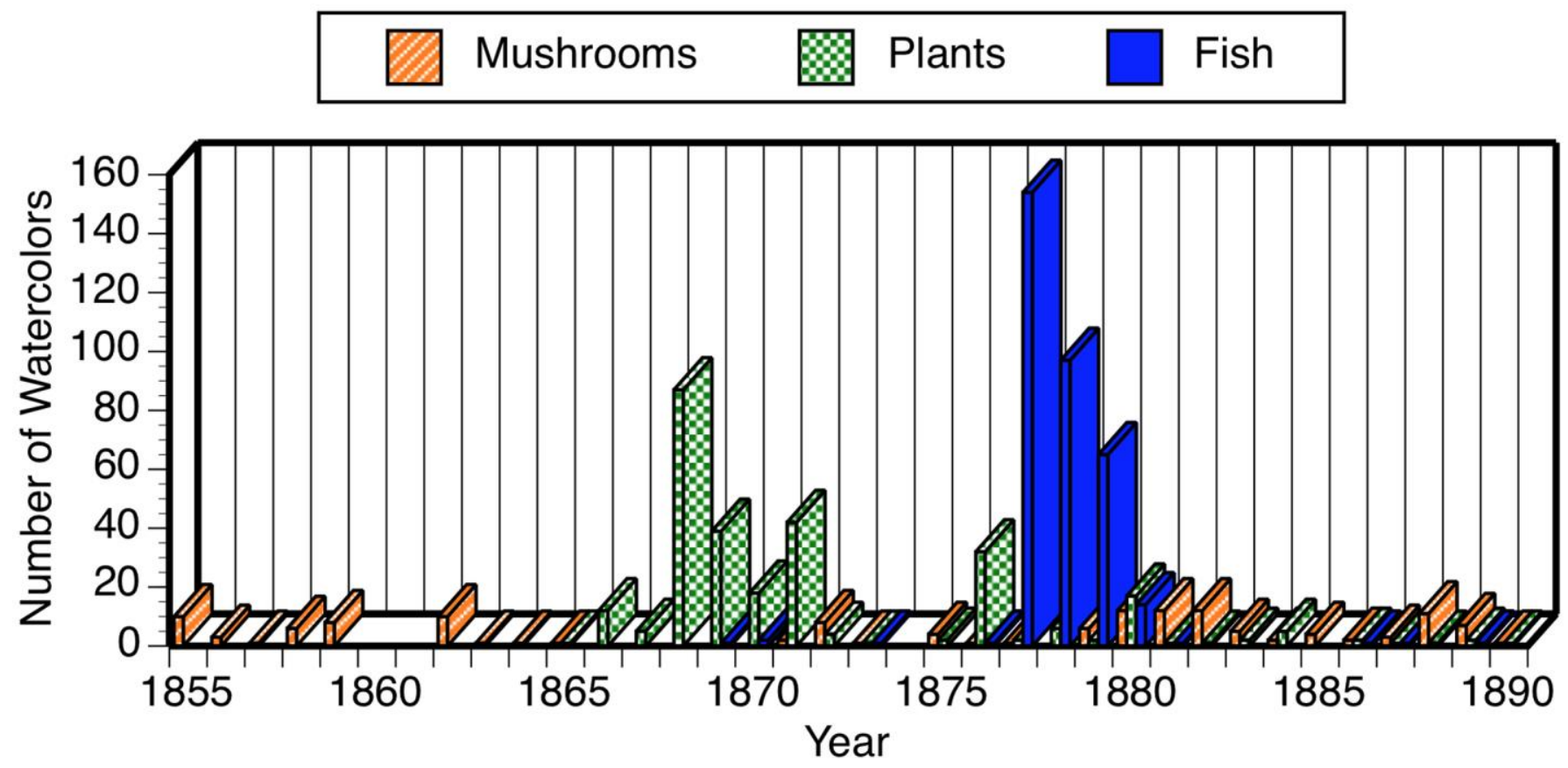

Fig. 5. Les sujets des aquarelles de Fossat ont varié avec le temps.

La production d'aquarelles de poissons de Fossat était vraiment remarquable. Il n'existe aucun compte rendu contemporain de ses méthodes. Le plus ancien semble être un article de 1914 sur la première exposition de ses aquarelles de poissons au Muséum d'Histoire Naturelle de Nice en décembre 1913, peu de temps après leur «redécouverte» par Caziot (Lavagna 1914). L'article précise que Fossat s'est rendu sur la plage pour rencontrer les pêcheurs qui ramenaient à terre leur prise du jour, encore vivante. Sur place, Fossat peignait ensuite d'après nature. Malheureusement, la fidélité de l'article peut être mise en doute car il indique que la date de décès de Fossat est 1871, soit 20 ans avant sa mort réelle. Néanmoins, l'image de Fossat peignant des poissons fraîchement pêchés sur la plage est indéniablement attrayante. La vue d'une telle scène par l'artiste Franck Rainaut est présentée dans la Fig. 6. Cependant, il est difficile d'imaginer que Fossat ait pu réaliser sur place ses aquarelles comportant de fins détails anatomiques. De plus, certaines aquarelles montrent plusieurs spécimens collectés à des dates différentes. Il aurait donc dû apporter sur le rivage la peinture du premier spécimen, dans l'espoir d'en collecter un autre. Il est probable que le « travail de terrain » de Fossat consistait simplement à enregistrer les couleurs et l'aspect général du poisson vivant et que la peinture finale, détaillée, était réalisée une fois de retour sur le rivage au Muséum avec le spécimen en main. À cet égard, il convient de souligner que Fossat notait sur ses tableaux la date de collecte du spécimen représenté, et non la date de la peinture. On peut supposer que la peinture a été réalisée peu de temps après le prélèvement du spécimen, avec un poisson frais pour ainsi dire, car les poissons non conservés, ou même conservés dans l'alcool, sont généralement déformés. 


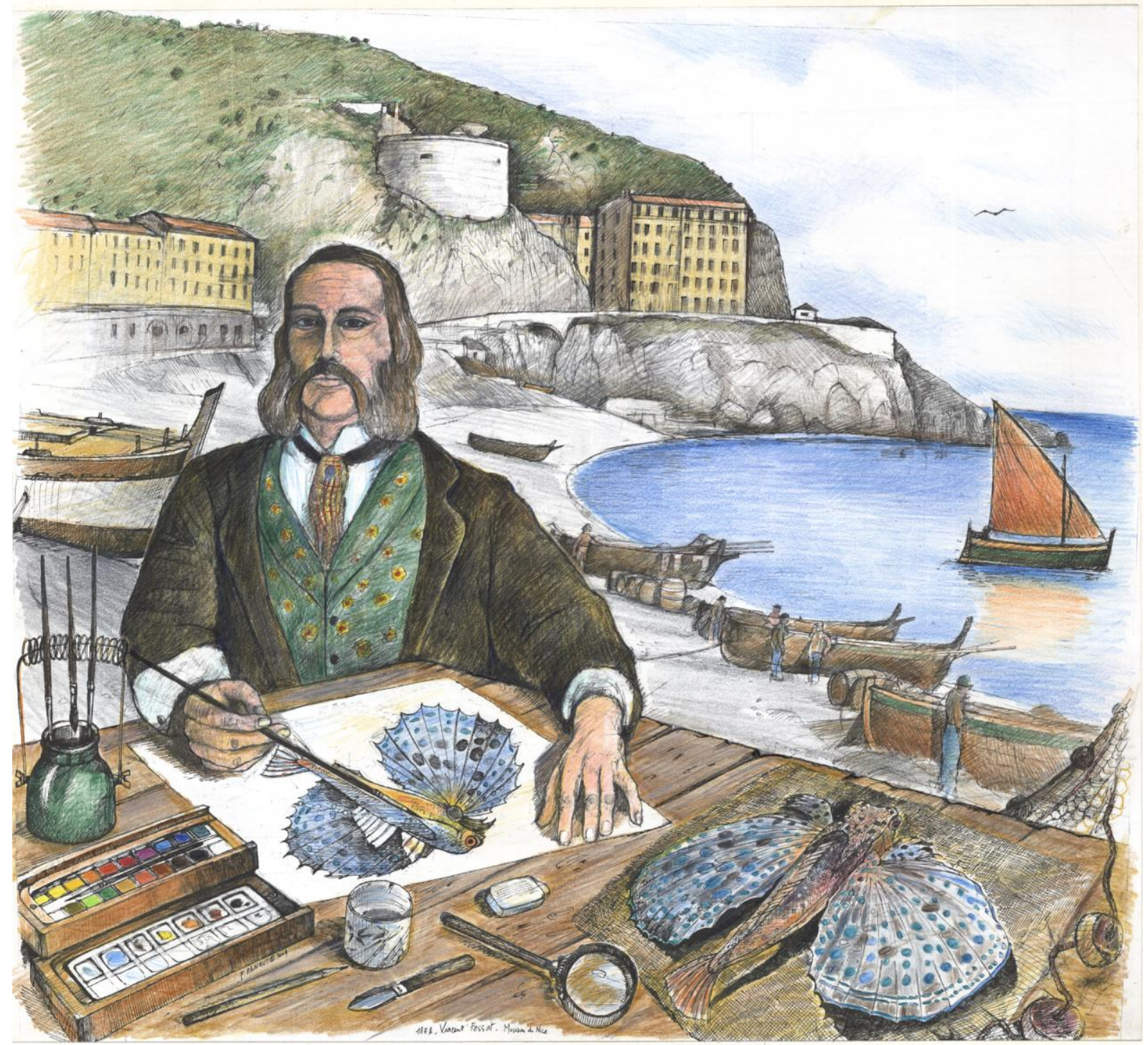

Fig. 6. "Vincent Fossat sur la plage des Ponchettes en 1878" par Frank Rainaut. La taille de l'original : 55 × $51 \mathrm{~cm}$. Tous droits réservés par Frank Rainaut.

Quel que soit le lieu où Fossat peignait ses aquarelles de poissons, sa production était parfois prodigieuse. Un grand nombre d'aquarelles ont été réalisées à partir de spécimens collectés au cours du seul mois de mai 1877. La figure 7 montre le nombre de spécimens collectés chaque jour du mois de mai, qui ont finalement été représentés dans une aquarelle. Il est clair qu'il aurait pu collecter davantage de spécimens, mais nous ne le saurons jamais. Quoi qu'il en soit, Fossat a collecté des spécimens presque tous les jours du mois de mai, ce qui montre qu'il rendait souvent visite au pêcheur. Si Fossat a peint son aquarelle le jour même où le spécimen a été collecté, certains jours il aurait fait 3 aquarelles ! 


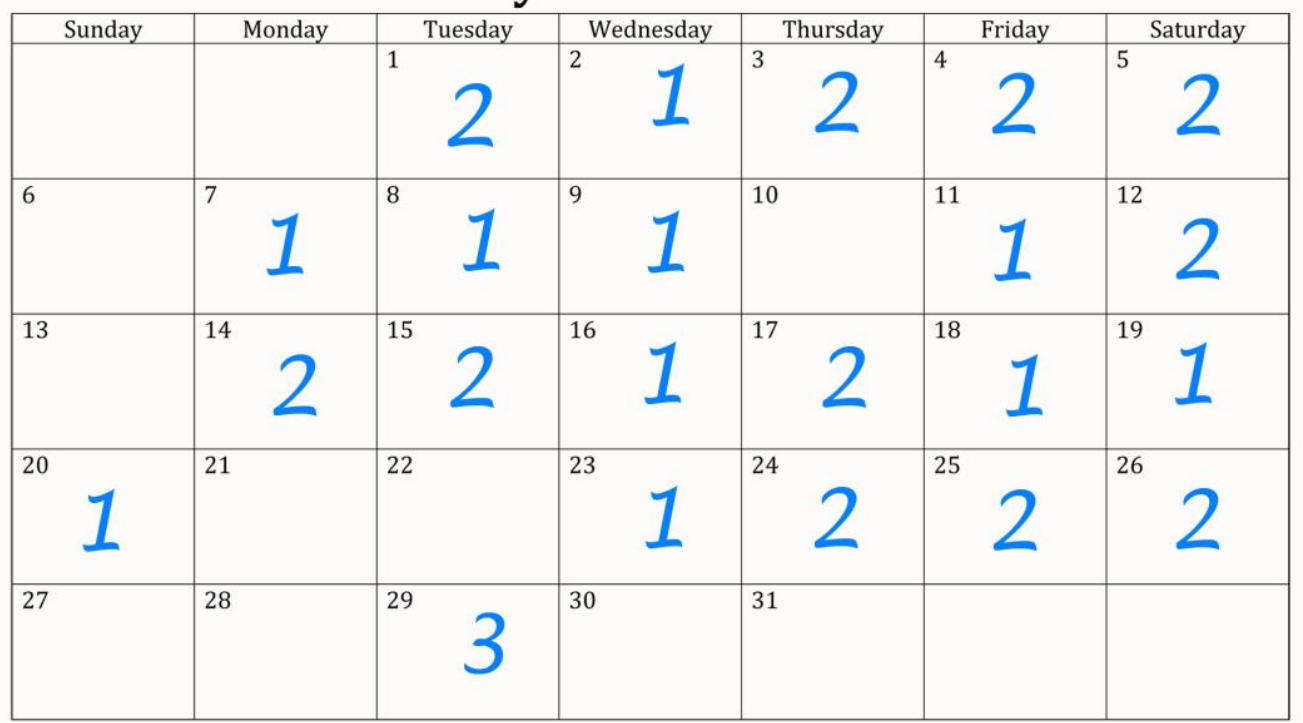

Fig 7. Le nombre de spécimens collectés chaque jour du mois de mai, qui ont finalement été représentés dans une aquarelle par Fossat.

Selon Jean Baptiste Barla, le travail de Vincent Fossat était essentiel.

Lorsque Fossat meurt en 1891, Barla écrit une lettre à la Revue Mycologique pour annoncer son décès (Anon, 1891). La lettre de Barla se serait terminée par : «Cette perte irréparable m'a plongé dans une affliction des plus extrêmes car Vincent Fossat était pour moi non seulement un collaborateur qui contribuait à enrichir mes collections de ses nombreuses illustrations mais il était également un ami fidèle et ancien ; aussi sa mort me prive-t-elle de tout espoir que j'avais de poursuivre mes travaux jusqu'à leur terme. Il serait presque impossible de trouver une autre personne ayant ses talents d'aquarelliste et de lithographe et je suis donc obligé de suspendre mon travail. ».

\section{L'art scientifique de Vincent Fossat}

Les 15 pages suivantes présentent un échantillon des aquarelles de Vincent Fossat.

Les œuvres ont été choisies pour démontrer l'ampleur taxonomique de ses sujets et la qualité remarquable de ses peintures. Il s'agit d'une sélection subjective parmi les 835 aquarelles qui peuvent être visualisées (bien qu'en petit format) sur le site Internet «Joconde » en utilisant le lien fourni ci-dessous.

La sélection proposée est dominée par les aquarelles de poissons et d'échinodermes de Fossat car ces illustrations inédites, contrairement à de nombreuses illustrations de champignons et d'orchidées, ne sont pas facilement accessibles dans les publications numérisées de Jean-Baptiste Barla. Des liens vers deux de ces publications sont fournis ci-dessous.

Champignons :

\section{https://books.google.fr/books?id=1hglAQAAMAAJ\&hl=fr\&source=gbs_navlinks_s}

Orchidées : 


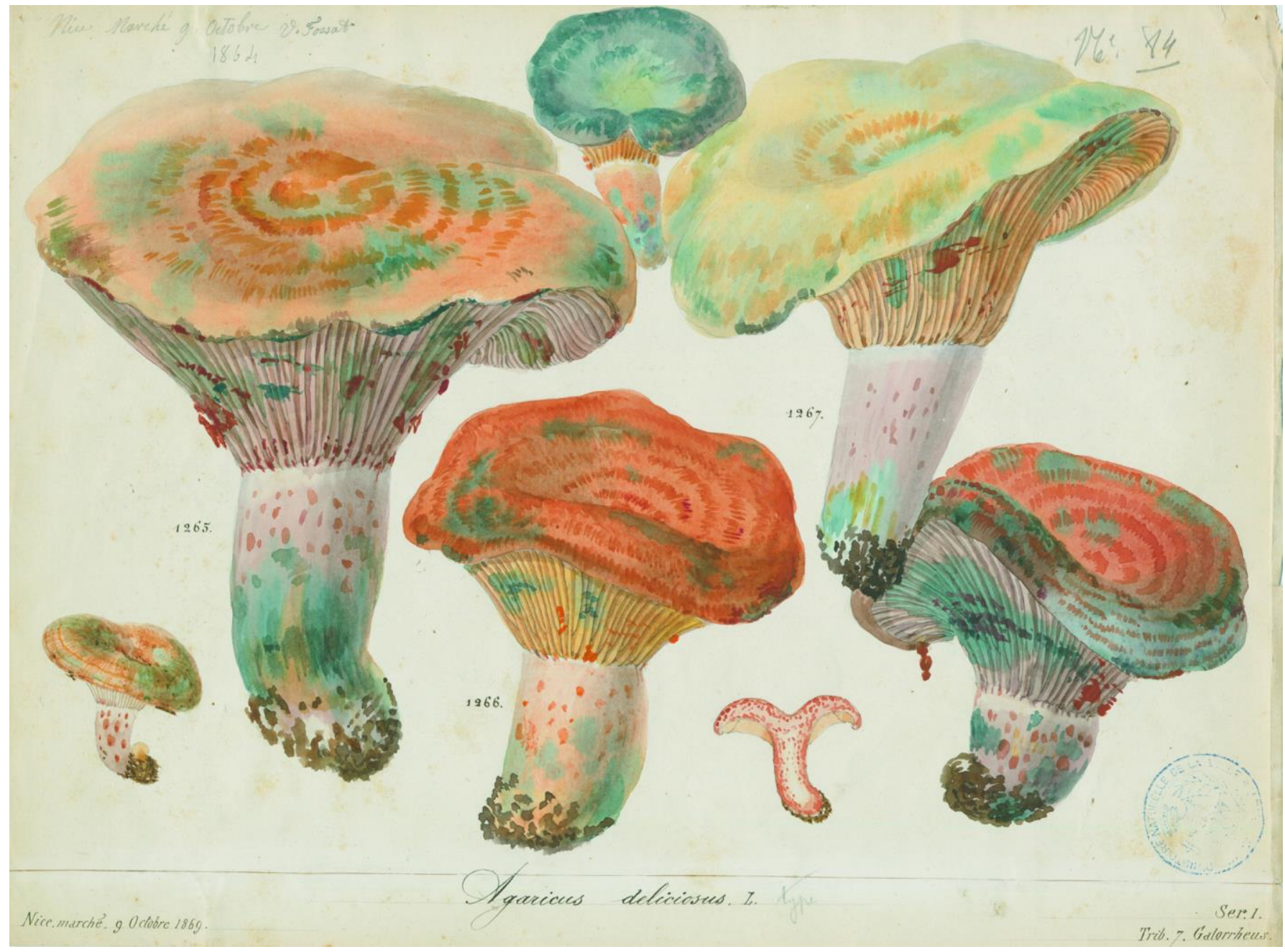

Fig. 8. Le champignon Lactarius deliciosus. La taille de l'original : $26,5 \mathrm{~cm} \times 35 \mathrm{~cm}$. 


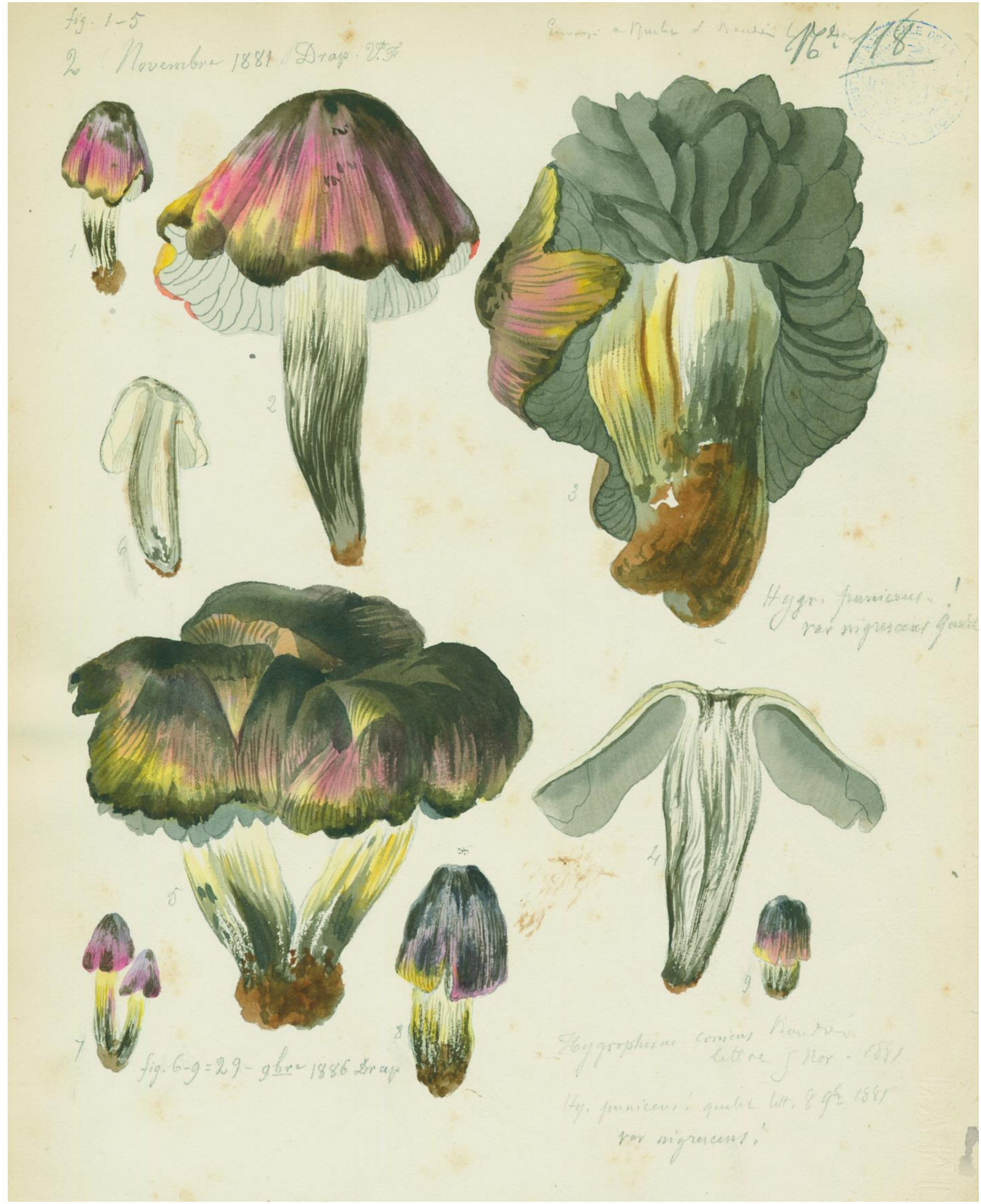

Fig. 9. Le champignon Hygrophorus conicus collected from Drap, a town inland from Nice, on November 02, 1881. La taille de l'original : $35 \mathrm{~cm} \times 27 \mathrm{~cm}$. 


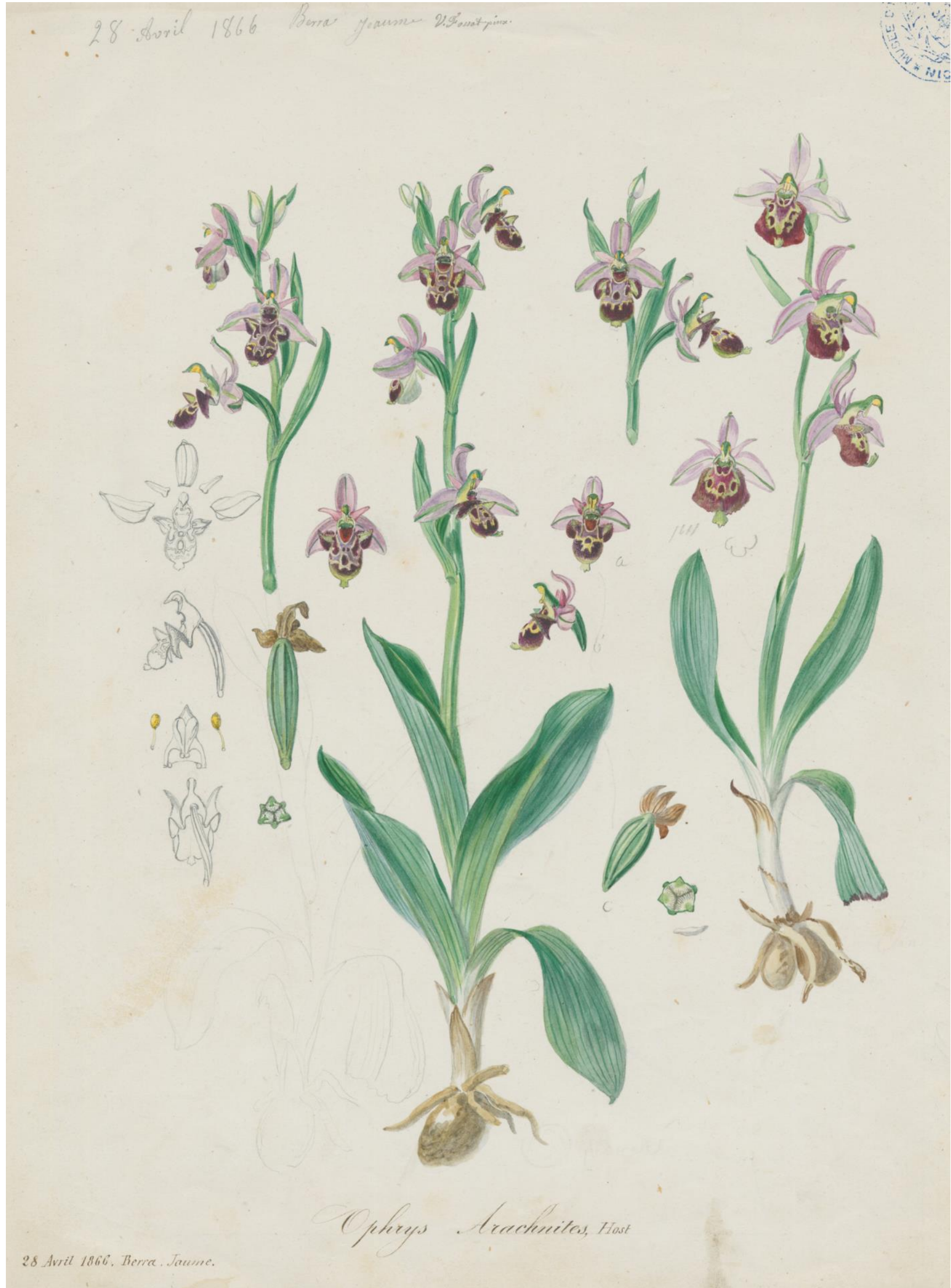

Fig. 10. L'orchidée Ophrys arachnites. La taille de l'original : $35,8 \mathrm{~cm} \times 27 \mathrm{~cm}$. 


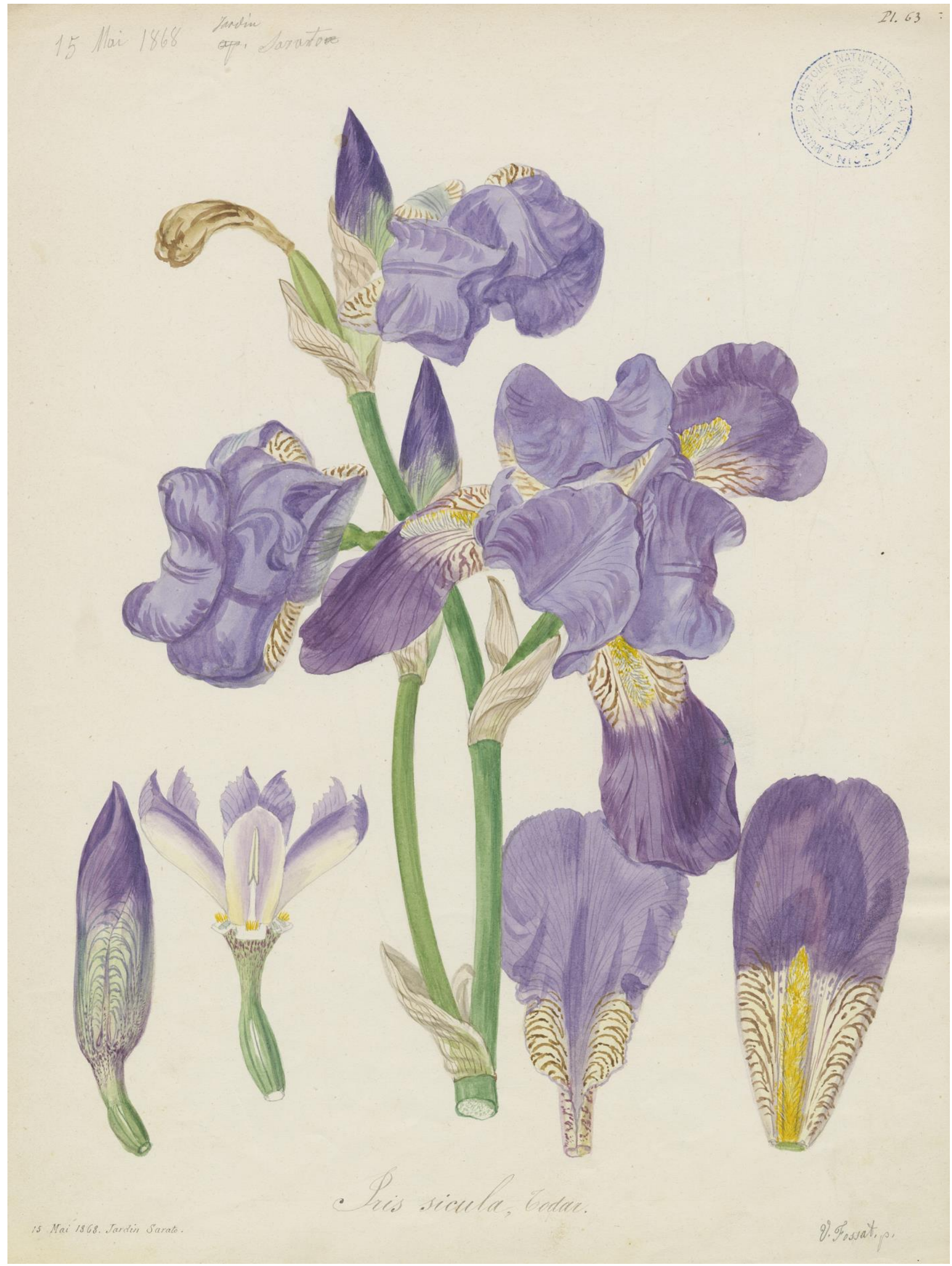

Fig. 11. L'iris Irus sicula. La taille de l'original : $35,8 \mathrm{~cm} \times 27 \mathrm{~cm}$. 


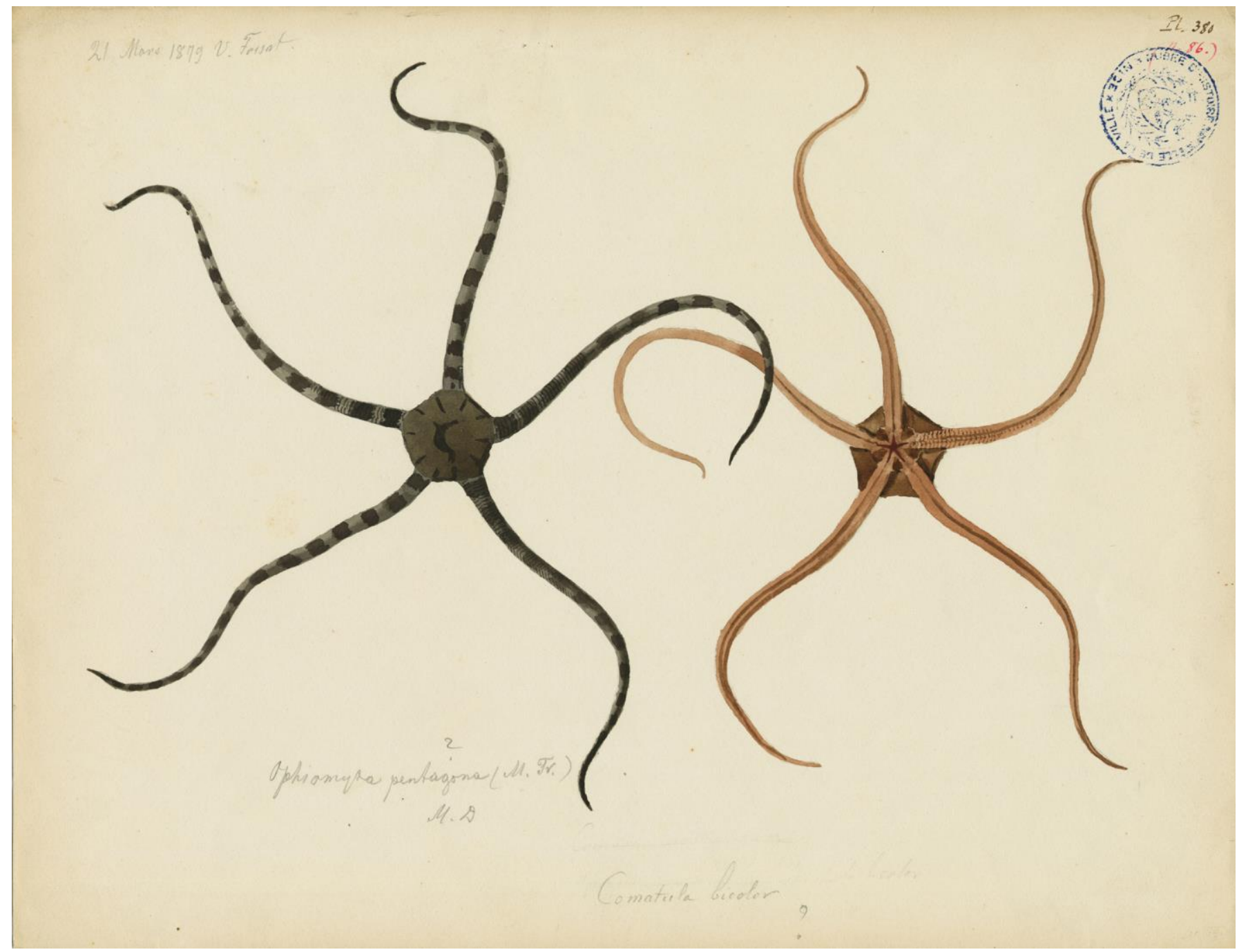

Fig. 12. L'étoile de mer Ophiomyta pentagona. La taille de l'original : $27 \mathrm{~cm}$ x 35,2 cm. 


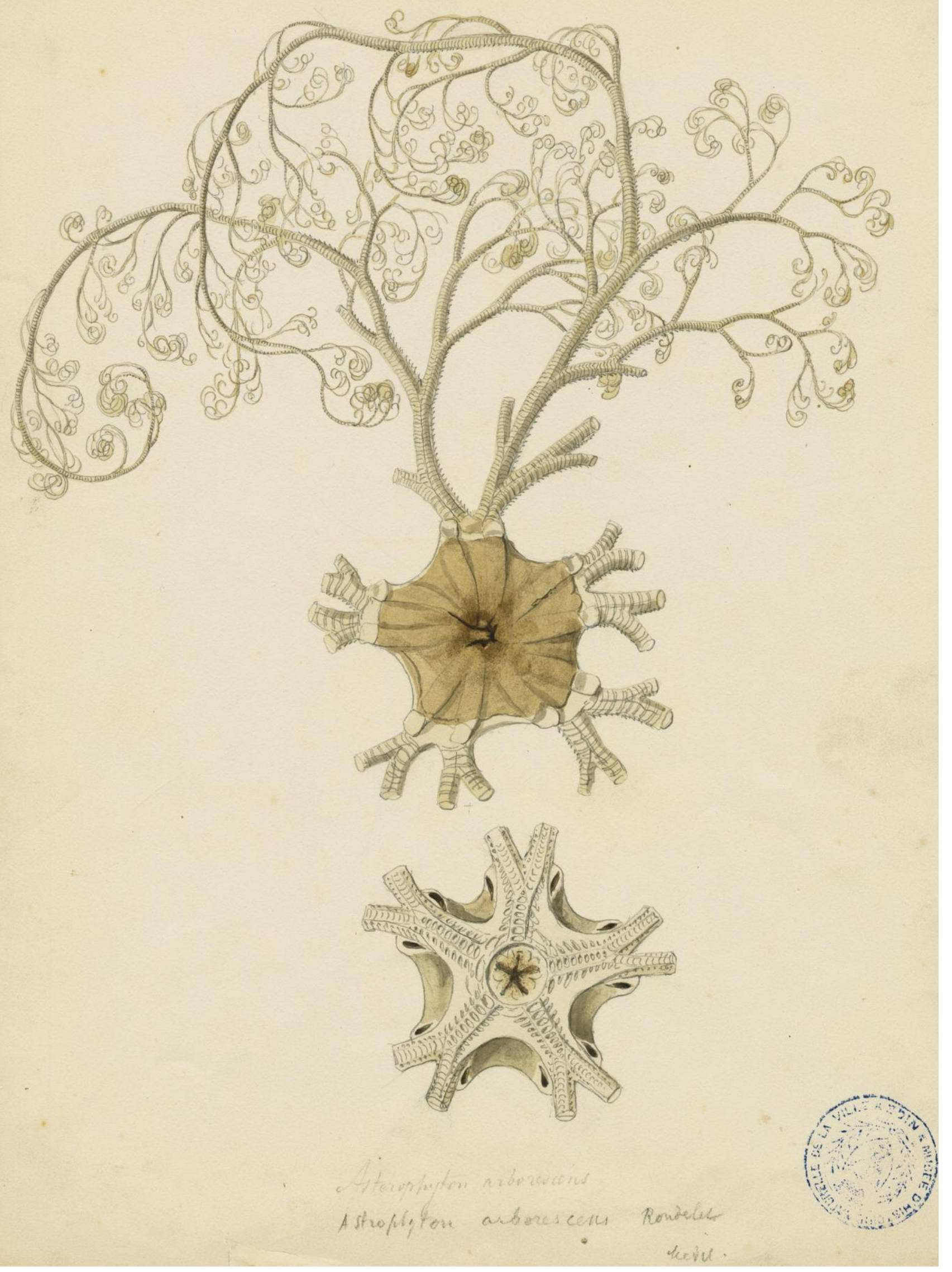

Fig. 13. L'étoile de mer Astrophyton arborescens. La taille de l'original : $35,3 \mathrm{~cm} \times 27 \mathrm{~cm}$. 


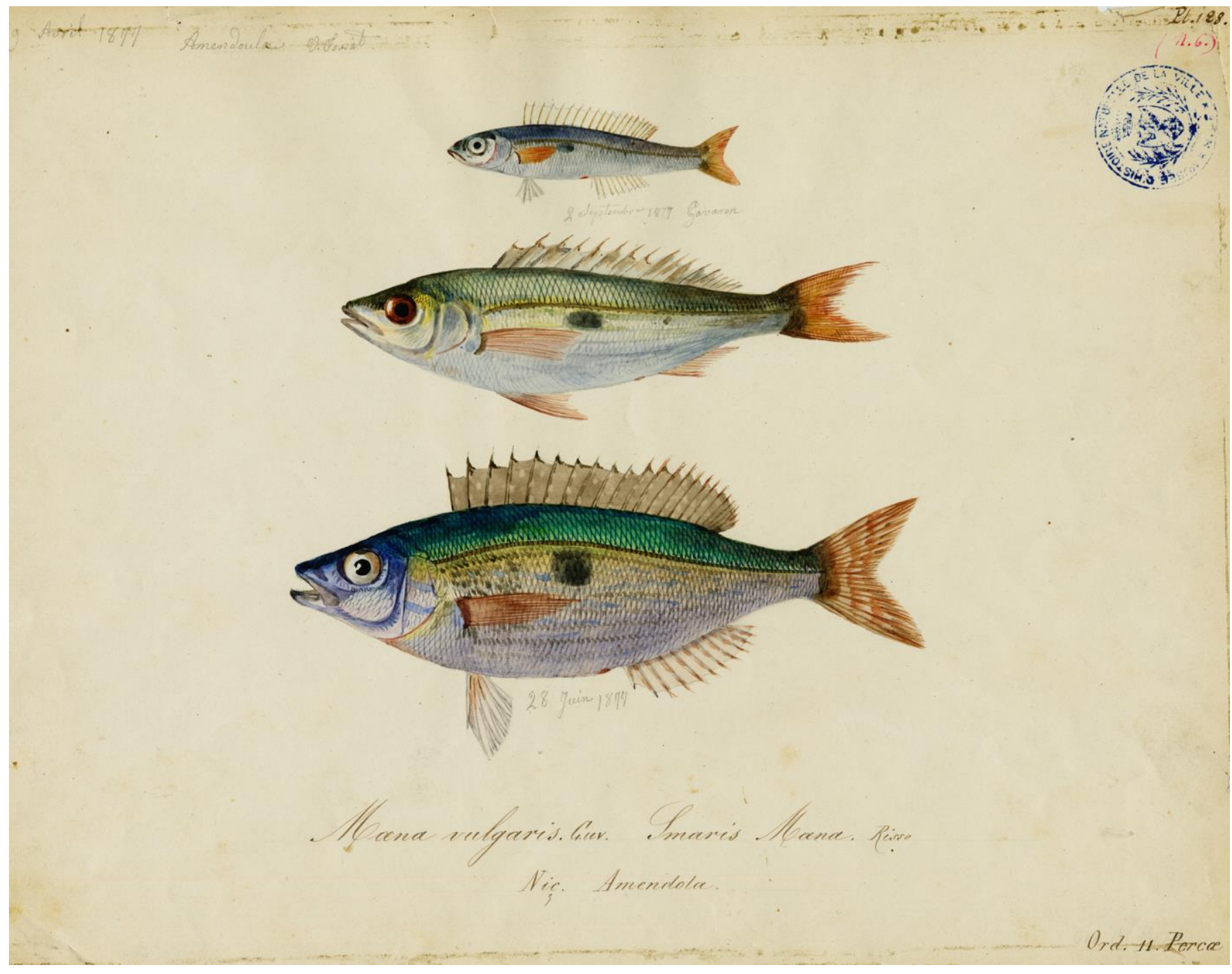

Fig. 14. Le poisson Spicara maena. La taille de l'original : $27 \mathrm{~cm} \times 35 \mathrm{~cm}$. 


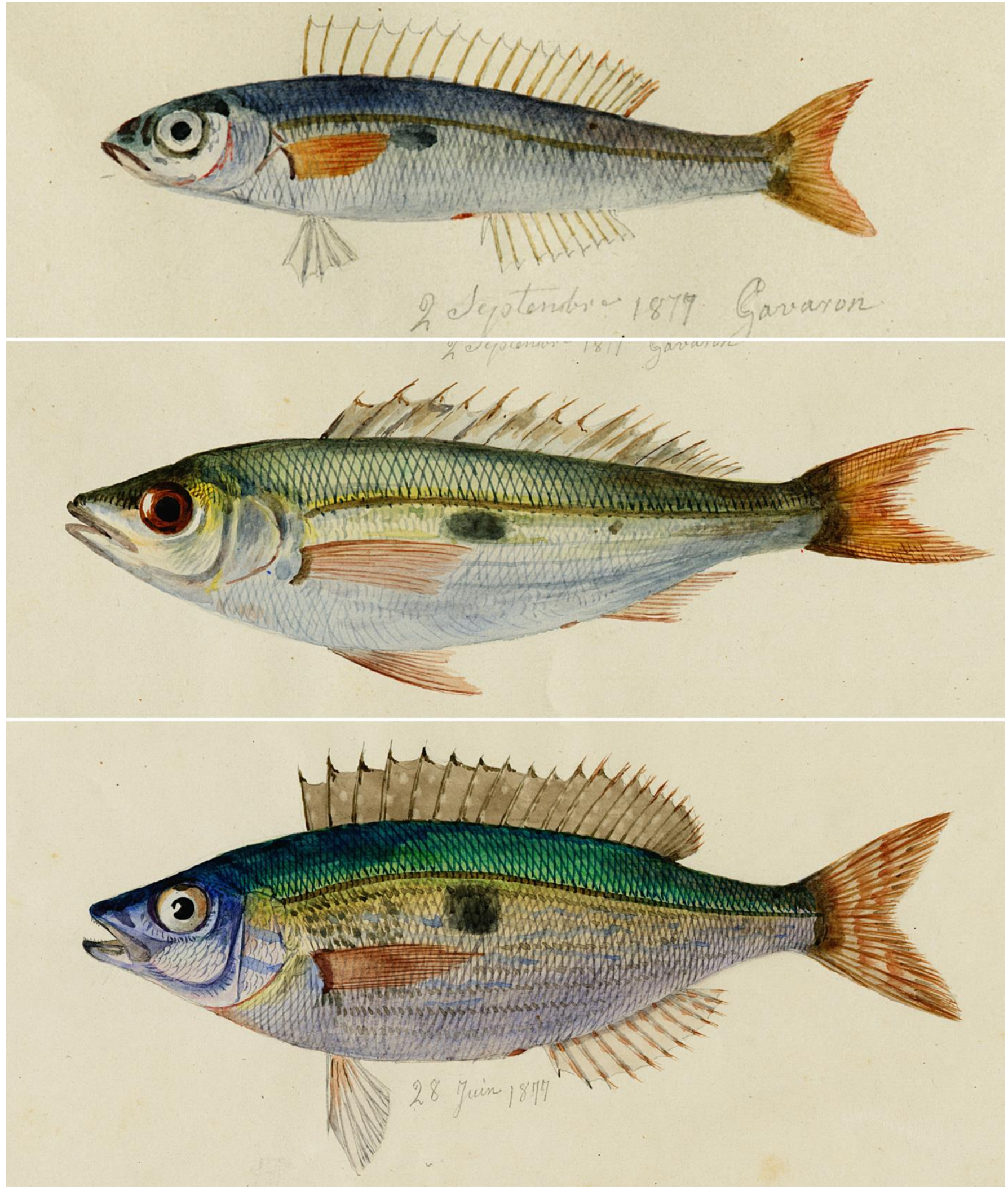

Fig. 15. Agrandissements des images du poisson Spicara maena. Les tailles des poissons dans l'original (Fig. 14), les longueurs, depuis le haut d'en bas, sont $8.1 \mathrm{~cm}, 15 \mathrm{~cm}$ et $18,3 \mathrm{~cm}$. 


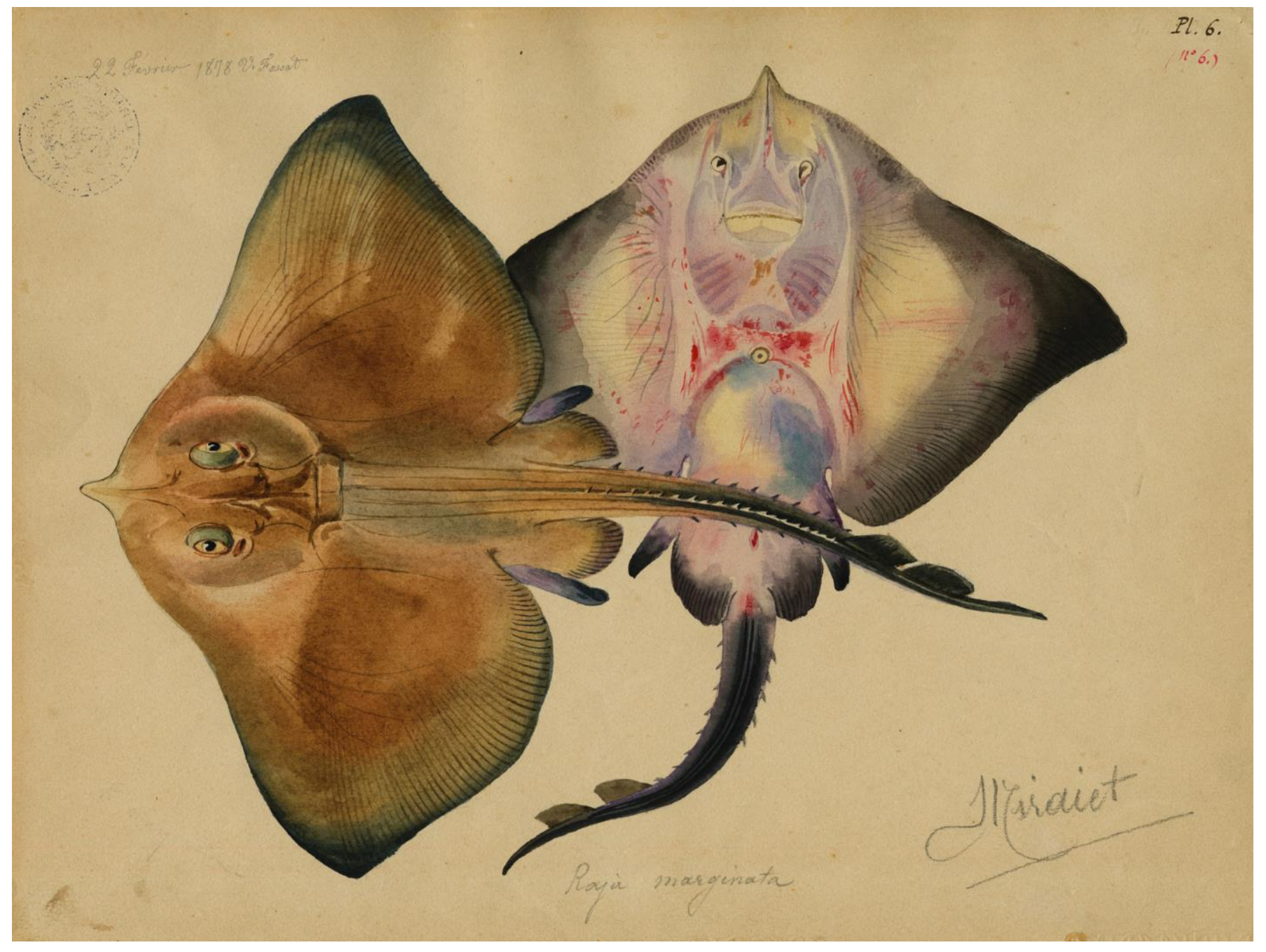

Fig. 16. Le poisson Raja alba. La taille de l'original : $26,8 \mathrm{~cm} \times 35 \mathrm{~cm}$. 


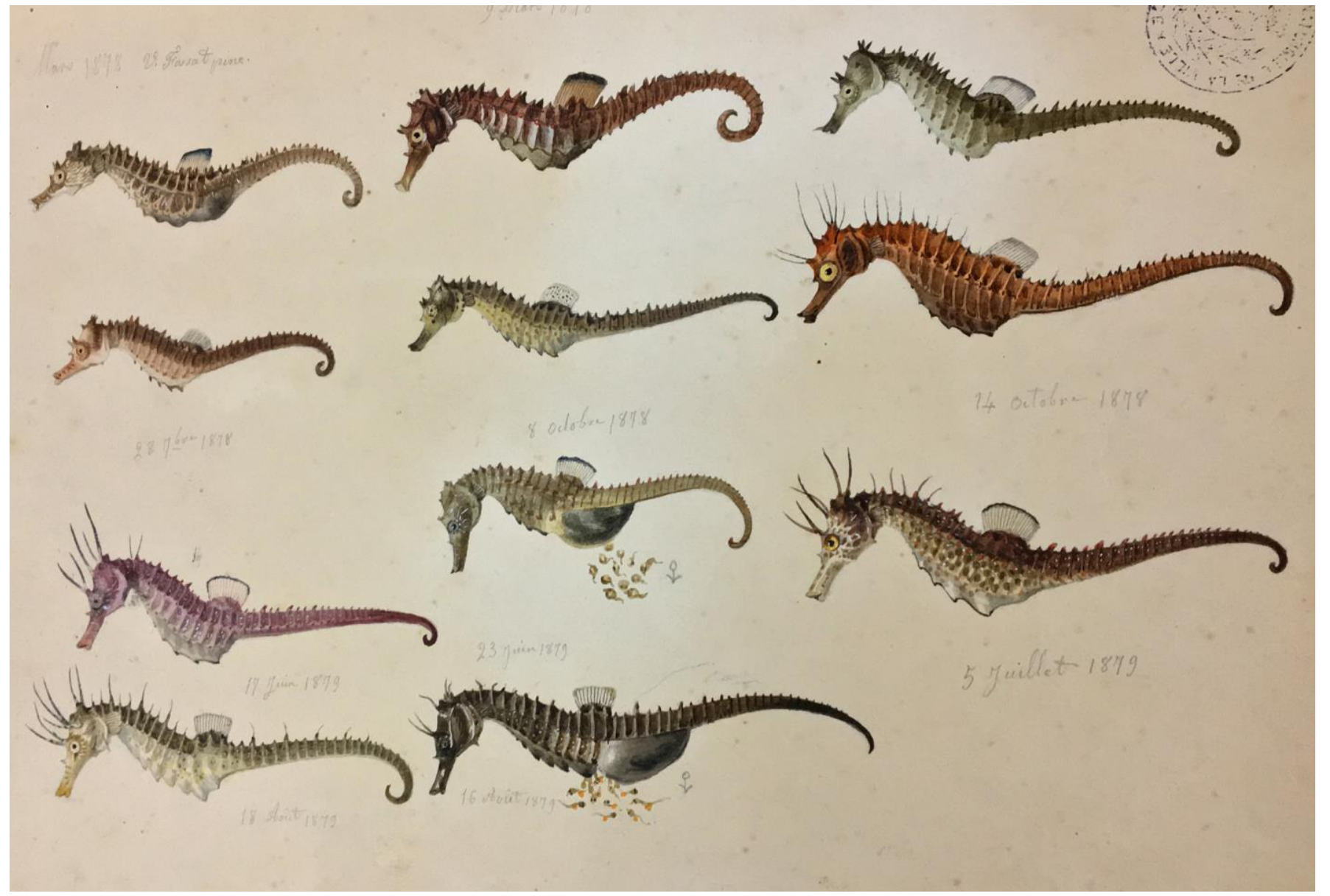

Fig. 17. Le poisson Hippocampus hippocampus collectés entre le 2 mars 1878 et le 23 juin 1879. La taille de l'original : $26,8 \mathrm{~cm} \times 33 \mathrm{~cm}$. 


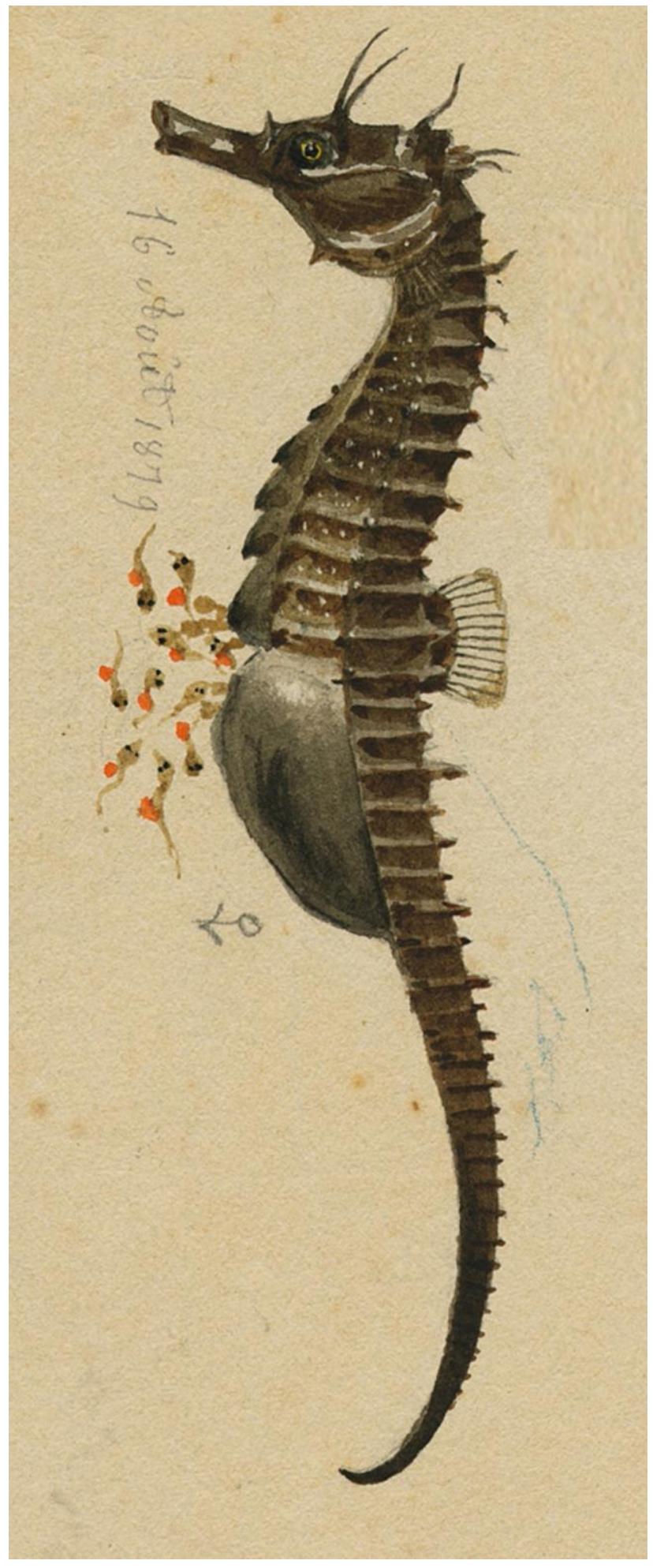

Fig. 18. Agrandissement de l'image du spécimen de Hippocampus hippocampus dans Fig. 16 Daté 16 octobre 1878. La taille de l'original (longueur) : 10,2 cm. 


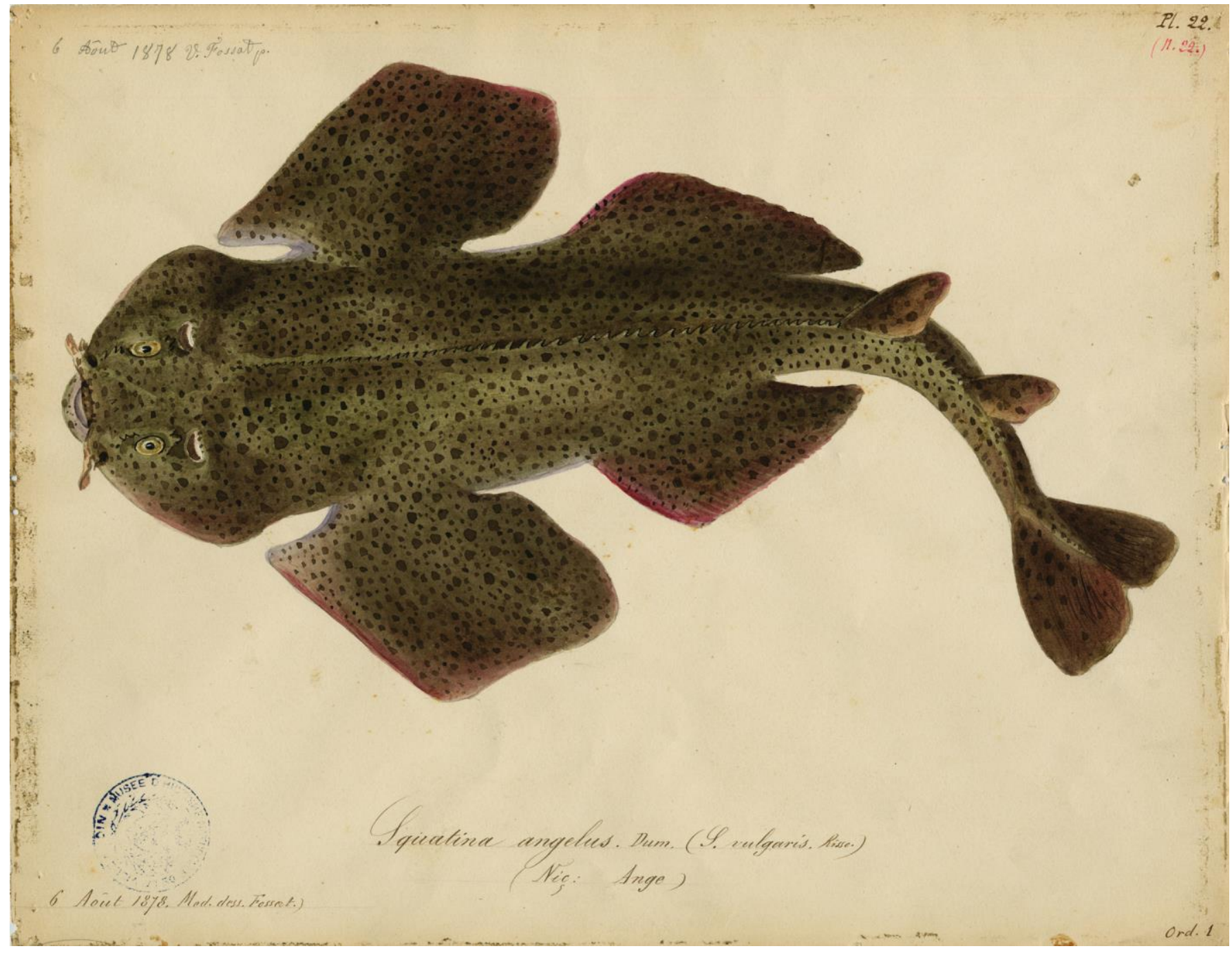

Fig. 19. Le poisson Squatina aculeata. La taille de l'original : $27,1 \mathrm{~cm} \times 35 \mathrm{~cm}$. 


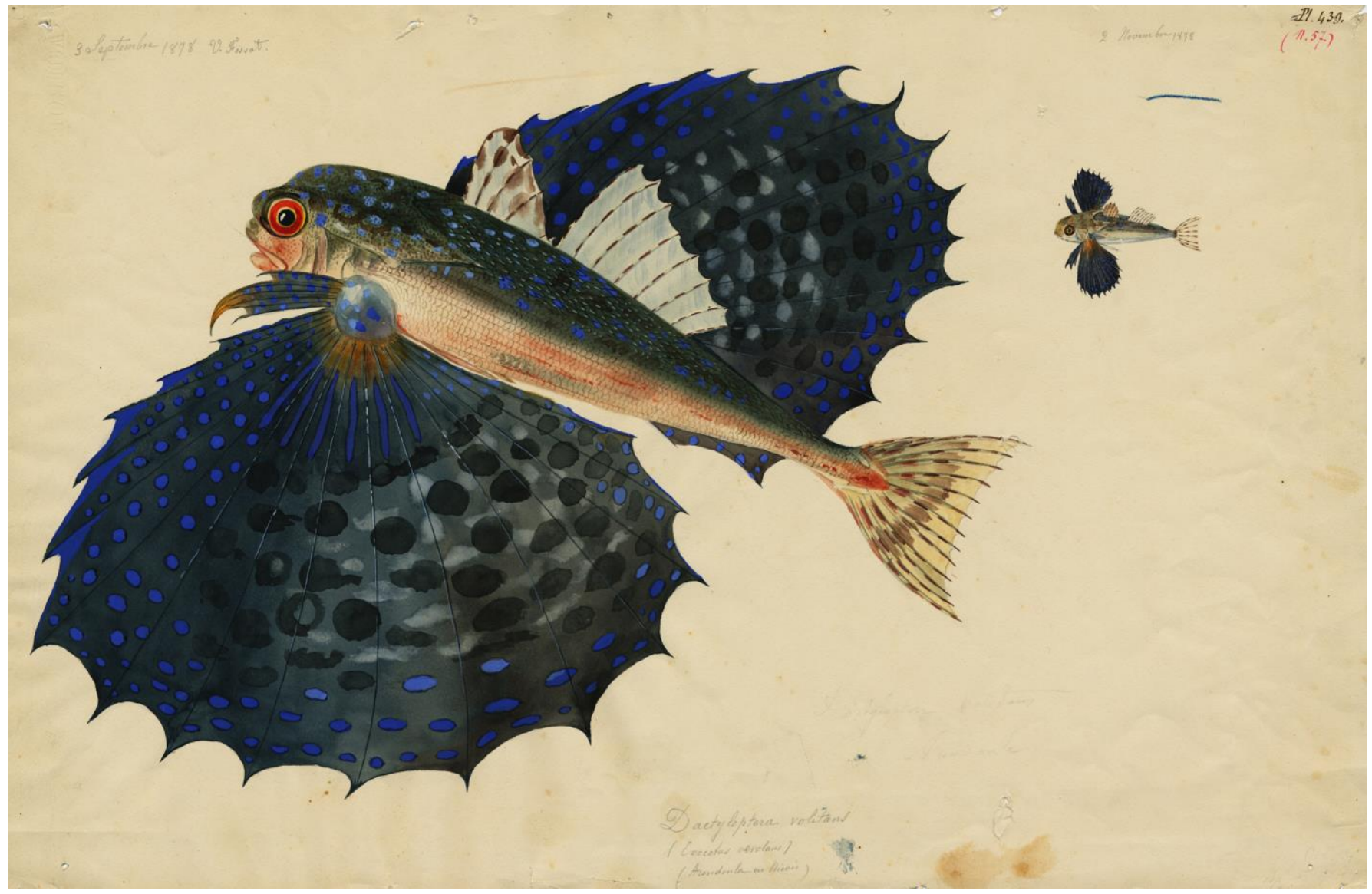

Fig. 20. Le poisson Dactylopterus volitans. La taille de l'original : $35,5 \mathrm{~cm}$ x $54 \mathrm{~cm}$. 


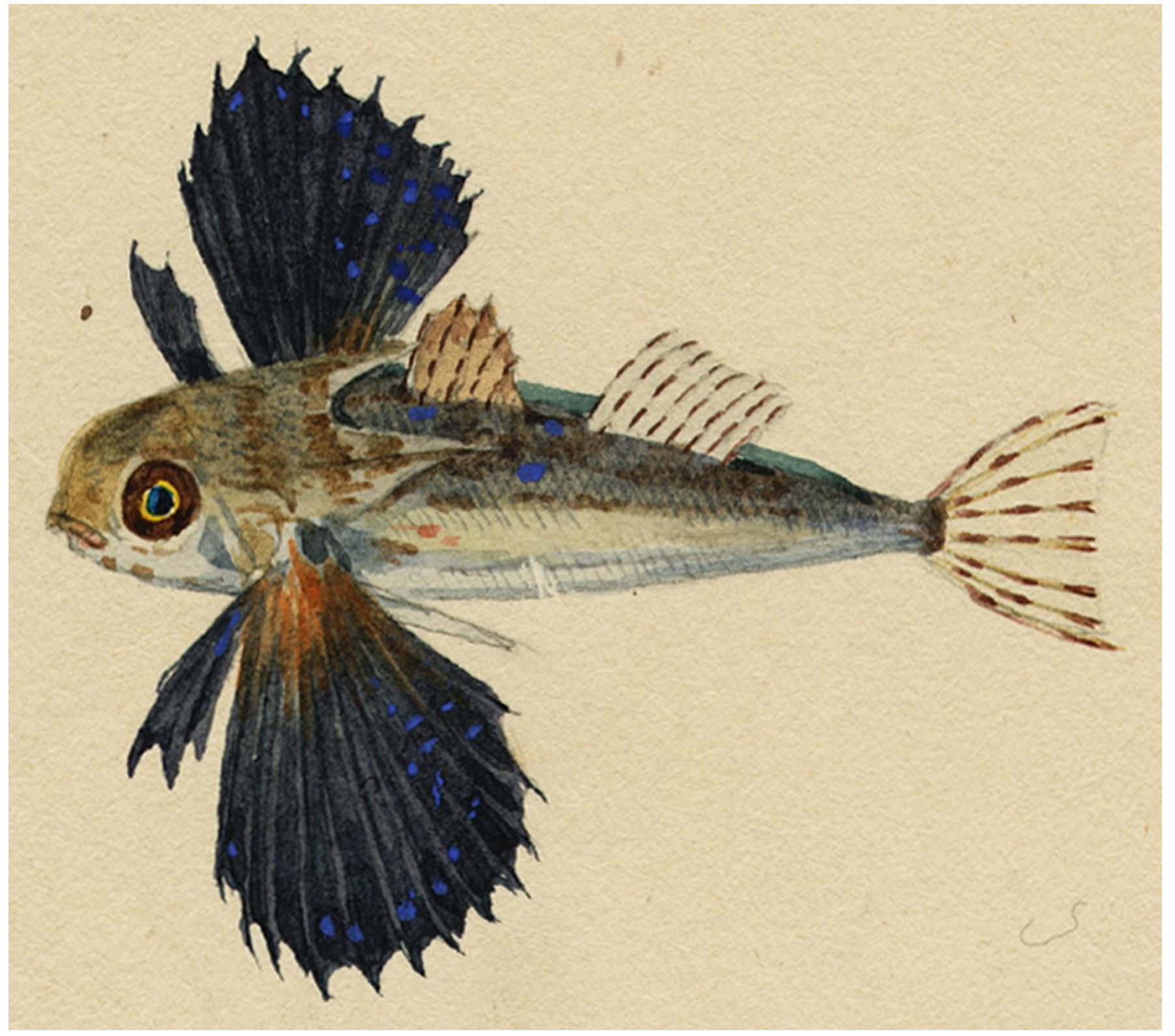

Fig. 21. Agrandissement de l'image du spécimen juvenile de Dactylopterus volitans dans figure 20. La taille (longuer) : $5,5 \mathrm{~cm}$. 


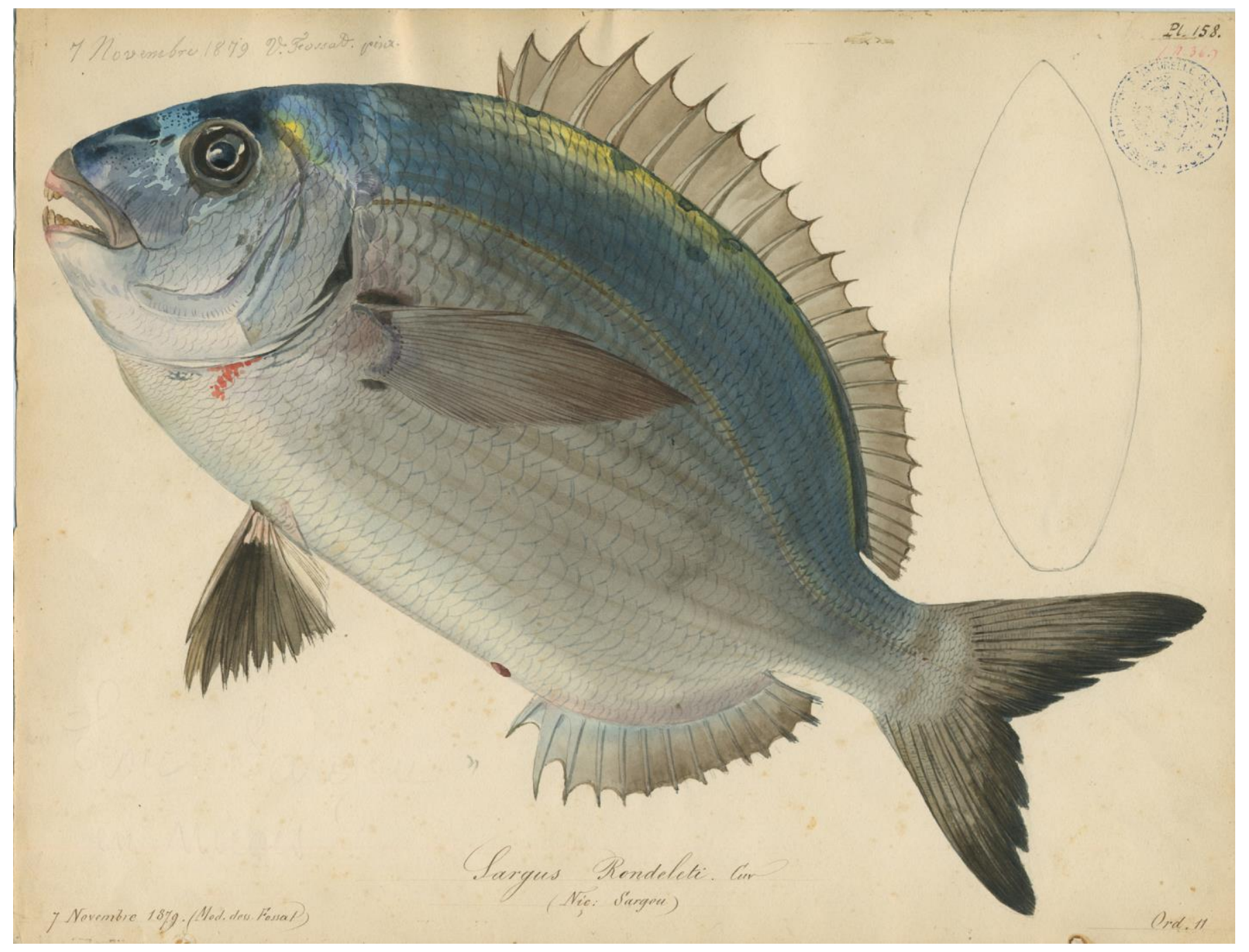

Fig. 22. Le poisson Diplodus sp. La taille de l'originale : $27 \mathrm{~cm} \times 35 \mathrm{~cm}$. 


\section{Remerciements}

Marie-Christine Maurel m'a invité à écrire cet article qu'elle a traduit à partir de ma version originale en anglais. Jean-Paul Potron, Julie Carraut, Elise Hansen, et Robin Cauvin du Musée Masséna ont fourni des informations et l'accès aux œuvres de Vincent Fossat. Franck Rainaut a permit le reproduction de son tableau. Joëlle Defaÿ, du Musée d'Histoire Naturelle de Nice, a fourni des aides précieuses. Sans son aide, cet article n'aurait pas pu être possible. Olivier Gerriet du Musée d'Histoire Naturelle de Nice a fourni des versions digitales des aquarelles de Vincent Fossat. Le Musée d'Histoire Naturelle de Nice est vivement remercié.

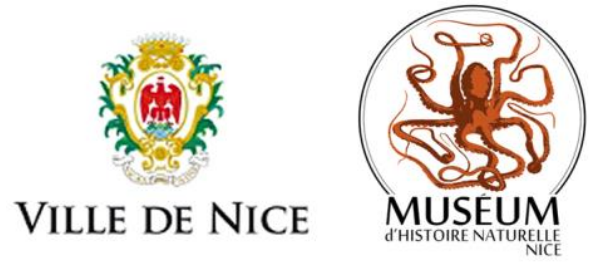

\section{Bibliographie}

Anon. 1891. Nouvelles. Revue Mycologique, 13th Yr., N 49, Jan. 1, 1891, pg 99.

Anon. 1923. Un hommage au peintre Vincent Fossat. Le Matin, noํ 12495, May 10, 1923: pg 4.

Bauchot, M.L.1996. Poissons de la mer de Nice peints par Vincent Fossat. Annales du Muséum d'Histoire Naturelle de Nice, 11:302-315.

Barla, J.-B. 1859. Les Champignons de la Province de Nice et Principlement les Especes Comestibles, Suspectes ou Vénéneuses Dessinés d'Apres Nature et Décrits. Nice: Imprimeries Canis Frères.

Barla, J.-B. 1868. Flore Illustrée de Nice et des Alpes-Maritimes. Iconographie des orchidées. Nice: Imprimerie Caisson et Mignon. https://gallica.bnf.fr/ark:/12148/bpt6k1025010n.texteImage

Barla, J.-B. 1876. Description et Figure du Xanthium Spinosum Lampourde Epineuse Spécifique contre l'Hydrophie. Nice: Imprimerie Caisson et Mignon.

Barla, J.-B. 1888. Flore Mycologique Illustrée les Champignins des Alpes-Martimes avec l'Indication de leurs propriétés utiles ou nuisibles. Nice: A. Gilletta.

Barla, J.-B. 1889a. Flore Mycologique Illustrée les Champignins des Alpes-Martimes aved l'Indication de leurs propriétés utiles ou nuisibles, 2, Gen. II. Lepiota.

Barla, J.-B. 1889b. Flore Mycologique Illustrée les Champignins des Alpes-Martimes aved l'Indication de leurs propriétés utiles ou nuisibles, 3 , Gen. III. Armilaria. Nice: A. Gilletta.

Barla, J.-B. 1890. Flore Mycologique Illustrée les Champignins des Alpes-Martimes aved l'Indication de leurs propriétés utiles ou nuisibles, 4 \& 5, Gen. IV. Tricholoma. Nice: A. Gilletta.

Barla, J.-B. 1892. Flore Mycologique Illustrée les Champignins des Alpes-Martimes aved l'Indication de leurs propriétés utiles ou nuisibles, 6, Gen. V. Clitocybe. Nice: A. Gilletta.

Beltrutti 1996a. Vincent Etienne Fossat 1822-1891. Annales du Muséum d'Histoire Naturelle de Nice, 11:51-54.

Beltrutti 1996b Biographie généalogique concernant Jean-Baptiste Barla. . Annales du Muséum d'Histoire Naturelle de Nice, 11:19-50.

Caziot, E. 1918. Notes biographiques de J.-B. Barla. Riviera Scientifique, Year 5, 113-125.

Chamagne Rollier, B., Defaÿ, J. 2013. Le Cabinet de Curiosités d'un Naturaliste du XIXe Siécle. Nice: Editions Gilletta Nice Matin.

Cuccuini, P. 2009. I funghi di Jean-Baptiste Barla, artista e naturalista. Il Museo di Storia Naturale dell'Università di Firenze, 2, 245-251.

Defaÿ, J. 1998. Vincent Fossat 1822-1891. in Acadèmia Nissarda, Le Pays de Nice et ses Peintres au XIXè Siecle. Nice: Acadèmia Nissardia.

Fighiera, E. 1912. Le peintre Louis Garneray à Nice. Nice Historique, 15th year, no. 3 (March), 117-123 
Fredj, G., Meinardi, M. 2007. L'Ange et l'Orchidée. Nice: Serre Editeur.

Isnard, P., Cordier, P. 1935. Le Musée d'Histoire Naturelle de Nice. La Terre et la Vie, 10:123-128.

Lavagna, J. 1914. Au musée d'histoire naturelle de Nice: Une exposition d'aquarelles. Riveria Scientifique, Year 1, January, 7-8.

Monot, J. 2011. Les Pêches Méditerranéenes, Voyage dans les Traditions. Versailles: Editions Quae.

Thevenon, L. 1990. Les musées de Nice (1925-1965). Nice Historique, year 1990, 110-120.

Trimbach, J. 1996. Barla et la mycologie. Annales du Muséum d'Histoire Naturelle de Nice, 11:179-256.

WWF/IUCN (2004). The Mediterranean deep-sea ecosystems: an overview of their diversity, structure, functioning and anthropogenic impacts, with a proposal for conservation. IUCN, Málaga and WWF, Rome. 